\title{
Finite element analysis of composites materials for aerospace applications
}

\begin{abstract}
Composites materials are intended to be used more extensively as an alternative of aluminum structure in aircraft and aerospace applications. This is due to their attractive properties as high strength-to-weight ratio and stiffness-to-weight ratio. Besides that it clarifies the growing interest for composites materials due to advantages of lightweight, high strength, high stiffness, superior fatigue life, tremendous corrosion resistance and low cost manufacturing. In this study, a finite element analysis (FEA) of fiberglass unidirectional Etype was analyzed in the framework of ABAQUS finite element commercial software. The analysis was done to quantify the mechanical properties and response of unidirectional Eglass in term of tensile, compression and thermal responses. From the analysis, the maximum and minimum values of stress and strain for E-glass 21xK43 Gevetex and Silenka E-glass 1200tex were obtained and stress-strain curve is presented. The ultimate load of failure, elastic behavior, tensile strength and other properties for each laminated plates under tensile and thermal-stress are determined from stress-strain curves. The simulation will run twice for each material where the first simulation based on orientation angles of $45^{\circ}$ for ply- $1,-45^{\circ}$ for ply- 2 and $90^{\circ}$ for ply-3 while the second simulation, the orientation angles is $0^{\circ}$ for all plies. The simulation is successfully conducted and verified by experimental data.
\end{abstract}

